Proceedings of the 363. WE-Heraeus Seminar on: "Neutron Stars and Pulsars" (Posters and contributed talks)

Physikzentrum Bad Honnef, Germany, May. 14-19, 2006, eds. W. Becker, H.H. Huang, MPE Report 291, pp. 201-204

\title{
Long term spectral variability in the soft gamma-ray repeater SGR $1900+14$
}

\author{
Paolo Esposito ${ }^{1,2}$, Sandro Mereghetti ${ }^{2}$, Andrea Tiengo ${ }^{2}$, Diego Götz ${ }^{3}$, Lara Sidoli ${ }^{2}$, and Marco Feroci ${ }^{4}$ \\ 1 Università di Pavia, Dipartimento di Fisica Nucleare e Teorica and INFN-Pavia, via Bassi 6, I-27100 Pavia, Italy \\ 2 INAF-IASF Milano, via Bassini 15, I-20133 Milan, Italy \\ 3 CEA Saclay, DSM/DAPNIA/Service d'Astrophysique, F-91191, Gif-sur-Yvette, France \\ 4 INAF-IASF Roma, via Fosso del Cavaliere 100, I-00133 Roma, Italy
}

\begin{abstract}
We present a systematic analysis of all the BeppoSAX data of SGR $1900+14$. The observations spanning five years show that the source was brighter than usual on two occasions: $\sim 20$ days after the August 1998 giant flare and during the $10^{5} \mathrm{~s}$ long $\mathrm{X}$-ray afterglow following the April 2001 intermediate flare. In the latter case, we explore the possibility of describing the observed short term softening only with a change of the temperature of a blackbody-like component. In the only BeppoSAX observation performed before the giant flare, the spectrum of the SGR 1900+14 persistent emission was significantly harder and possibly detected also above $10 \mathrm{keV}$ with the PDS instrument. In the last BeppoSAX observation (April 2002 ) the flux was $\sim 25 \%$ lower than the historical level, suggesting that the source was entering a quiescent period.
\end{abstract}

\section{Introduction}

The soft gamma-ray repeater (SGR) SGR 1900+14 was discovered in 1979 through series of short and soft gamma-ray bursts (Mazets et al. 1979). Many years later, its persistent pulsating $\mathrm{X}$-ray counterpart was discovered in the $2-10 \mathrm{keV}$ energy band (Hurley et al. 1999b). More recently, it was also detected in the hard X-ray range (20-100 keV) with the INTEGRAL satellite, becoming the second SGR, after SGR 1806-20, established as a persistent hard X-ray source (Götz et al. 2006).

The rather discontinuous bursting activity of SGR 1900+14 (see Fig. 1 bottom panel) raised to a summit on 1998 August 27 with the emission of a giant flare, when more than $10^{44}$ ergs of $\gamma$-rays were emitted in less than one second (Hurley et al. 1999a). This was one of the three giant flares detected up to now from three different SGRs. The extreme properties of these events are the main motivation for the magnetar interpretation. In this model (Thompson \& Duncan 1995, 1996), the SGRs and the Anomalous X-ray Pulsars (AXPs, another class of X-ray sources with similar properties, see e.g. Mereghetti et al. 2002) are believed to be neutron stars powered by the decay of their extremely intense magnetic
Table 1. Log of the BeppoSAX observations of SGR $1900+14$

\begin{tabular}{|c|c|c|}
\hline Obs & Date & Instrument / exposure \\
\hline A & 1997 May 12 & LECS / $20 \mathrm{ks}$ MECS / $46 \mathrm{ks}$ PDS / $20 \mathrm{ks}$ \\
\hline B & 1998 Sep 15 & LECS / 14 ks MECS / 33 ks PDS / 16 ks \\
\hline $\mathrm{C}$ & 2000 Mar 30 & LECS / 14 ks MECS / $40 \mathrm{ks}$ PDS / $18 \mathrm{ks}$ \\
\hline $\mathrm{D}$ & 2000 Apr 25 & LECS / 17 ks MECS / $40 \mathrm{ks}$ PDS / $19 \mathrm{ks}$ \\
\hline $\mathrm{E}$ & 2001 Apr 18 & LECS / $20 \mathrm{ks}$ MECS / $46 \mathrm{ks}$ PDS / $17 \mathrm{ks}$ \\
\hline $\mathrm{F}$ & 2001 Apr 29 & LECS / $26 \mathrm{ks}$ MECS / $58 \mathrm{ks}$ PDS / $26 \mathrm{ks}$ \\
\hline G & 2002 Mar 09 & $\mathrm{PDS} / 48 \mathrm{ks}$ \\
\hline $\mathrm{H}$ & 2002 Apr 27 & MECS / $83 \mathrm{ks}$ \\
\hline
\end{tabular}

field $\left(B \sim 10^{14}-10^{15} \mathrm{G}\right)$ rather than by rotation.

Here we present the analysis of the persistent emission of SGR $1900+14$ by means of the BeppoSAX satellite, both in the soft and hard $\mathrm{X}$-ray range, and its evolution across the giant flare and in relation to its bursting activity.

\section{Soft X-ray emission}

\subsection{Observations and data analysis}

We have analyzed all the X-ray observations of SGR 1900+14 performed with the BeppoSAX satellite (see Table (1). The spectra were extracted from the MECS (Boella et al. 1997) and LECS (Parmar et al. 1997) instruments using circles with radii $4^{\prime}$ and $8^{\prime}$, respectively. The background spectra were extracted in all cases from nearby regions and time filters were applied to both the source and background spectra to exclude the SGR bursts detected during observation $\mathrm{B}, \mathrm{E}$ and $\mathrm{F}$.

\subsection{Spectral results}

We have first tried to fit the spectra with an absorbed power-law model, but three observations give unacceptable values of the $\chi^{2}$ and structured residuals. For 


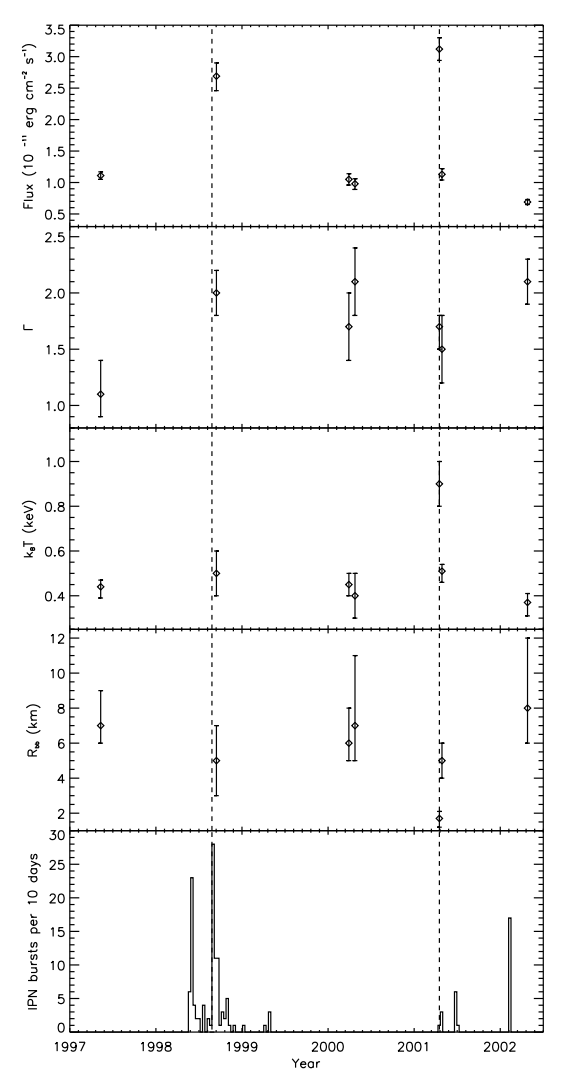

Fig. 1. Long term evolution of the $2-10 \mathrm{keV}$ unabsorbed flux, the spectral parameters (for an absorbed power-law plus blackbody model, assuming $n_{\mathrm{H}}=2.6 \times 10^{22} \mathrm{~cm}^{-2}$ ) and the burst activity (as observed by the Interplanetary Network) of SGR $1900+14$. The vertical dashed lines indicate the giant and intermediate flares (1998 August 27 and 2001 April 18, respectively).

these observations, good fits are obtained with the addition of a blackbody component. Since such a twocomponents model is typical of the magnetar candidates (Woods \& Thompson 2004), we have used this model to fit all the available spectra, obtaining the results reported in Fig. 1. The blackbody parameters are compatible in all the available observations, except for that taken during the afterglow of the intermediate flare (observation E). This indicates that a constant blackbody component with $k_{B} T \sim 0.4 \mathrm{keV}$ and emitting area with $R \sim 6-7 \mathrm{~km}$, might be a permanent feature of the X-ray spectrum of SGR $1900+14$.

As can be seen in the upper panel of Fig. 1, the flux varies by a factor $>5$, with the highest values observed during observations B and E. These two observations were taken shortly after extreme bursting events. The former was performed 20 days after the giant flare, that was followed by a $\sim 2$ months period of enhanced X-ray flux (Woods et al. 1999). The latter started only 7.5 hours after the intermediate flare which had a fluence

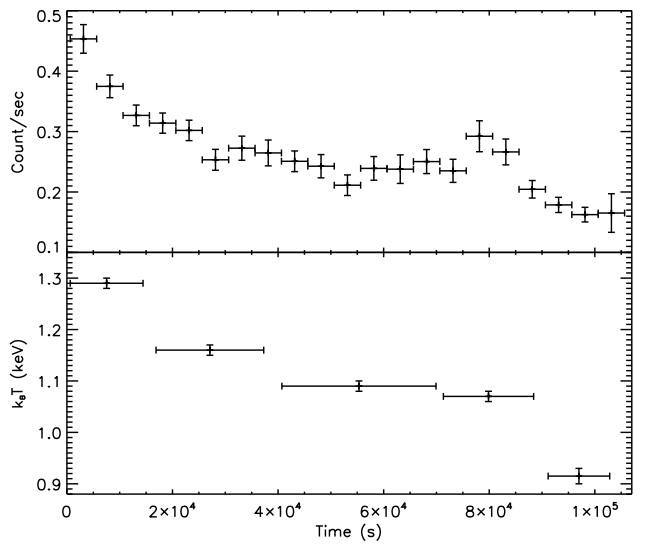

Fig. 2. Background subtracted MECS 2-10 keV light curve and blackbody temperature observed on 2001 April 18 about 7.5 hours after the flare. The latter values correspond to an additional blackbody component that can be interpreted as due to a portion of the neutron star surface heated during the flare (see Section 2.2).

$\sim 20$ times lower than that of the 2001 April 18 giant flare (Guidorzi et al. 2004).

The afterglow following this bright burst is clearly visible during the BeppoSAX observation as a decrease in the X-ray flux (see Fig. 2, top panel), accompanied by a significant softening of the spectrum (Feroci et al. 2003). In addition to the afterglow analysis already reported by Feroci et al. (2003), we have performed a time resolved spectroscopy of the afterglow by dividing observation $\mathrm{E}$ into five time intervals. Under the assumption that the variable "afterglow" emission is present on top of a "quiescent" emission that shows only moderate variations on long time-scales, we fitted them with a model consisting of a power-law plus blackbody with fixed parameters (as representative parameters of the fixed quiescent emission we used values consistent with those seen in the last observations before the flare: $\mathrm{C}$ and $\mathrm{D}$ ), plus a third variable component to model the afterglow emission. Although the "afterglow emission" in the five spectra can be fitted by a variety of models, the spectral evolution of the afterglow is well represented by an additional blackbody component with fixed emitting area (radius of $\sim 1.5 \mathrm{~km}$, for a source distance of $15 \mathrm{kpc}$ ) and progressively decreasing temperature $\left(k_{B} T\right.$ from $\sim 1.3$ to $\sim 0.9 \mathrm{keV}$, see Fig. 2 , bottom panel), that can be interpreted as due to a portion of the neutron star surface heated during the flare.

Excluding the two observations taken after the exceptional explosive events (B and E), the flux of SGR 1900+14 had a rather constant value of $\sim 10^{-11} \mathrm{erg} \mathrm{cm}^{-2} \mathrm{~s}^{-1}$ from 1997 to 2001. On the other hand a significantly lower flux level was seen in the following observations. The flux decrease actually started when the source was still moderately active (the flux in observation $\mathrm{H}$ is at least $\sim 20 \%$ lower than in all the previous quiescent observations) and 
has been interrupted by a slight rise in coincidence with the March 2006 burst reactivation, as shown by recent XMM-Newton observations (Mereghetti et al. 2006).

Although the flux of the only pre-giant flare observation is compatible with that of the quiescent post-flare observations taken before 2002, its spectrum is significantly harder, as shown in the second panel of Fig. 1 The overall hardness of the pre-flare observation is confirmed by the fact that the spectra C, D, F, and $\mathrm{H}$ can be simultaneously fit with the same parameters (introducing a normalization factor to account for the flux change), while the addition of spectrum A gives an unacceptable fit with structured residuals.

\section{Hard X-ray emission}

\subsection{Detection with the PDS instrument}

To study the high energy emission from SGR $1900+14$ we used the BeppoSAX PDS instrument Frontera et al. 1997), which operated in the $15-300 \mathrm{keV}$ range. The PDS instrument was more sensitive than INTEGRAL in this energy band, but it had no imaging capabilities and therefore the possible contamination from nearby sources must be taken into account. The field of view of the PDS instrument was $1.3^{\circ}$ (FWHM) and the background subtraction was performed through a rocking system that pointed to two $3.5^{\circ}$ offset positions every $96 \mathrm{~s}$.

In the case of SGR $1900+14$, the background pointings were free of contaminating sources, as confirmed by the identical count rates observed in the two offset positions during each observation. The field of SGR $1900+14$ is instead rather crowded, with three transient sources: the X-ray pulsars 4U 1907+97 (Liu et al. 2000) and XTE J1906+09 (Liu et al.|2000), and the black hole candidate XTE J1908+94 (in't Zand et al. 2002), located at angular distances of $47^{\prime}, 33^{\prime}$ and $24^{\prime}$ from the SGR, respectively. The pulsations of the two pulsars are clearly visible in the PDS data below $50 \mathrm{keV}$ when they are active, while XTE J1908+94, if in outburst, is clearly visible in the simultaneous MECS and LECS images and, being very bright, also in the lightcurve collected by the All Sky Monitor (ASM) on board the RossiXTE satellite. We have found that at least one of these contaminating sources was on in all the BeppoSAX observations except for the first one (see Table 2). Thus, only the 1997 observation (observation A), during which a significant signal was detected in the background subtracted PDS data, can be used to study SGR 1900+14 without the problem of contaminating sources. We searched for the SGR pulsation period ( $5.15719 \mathrm{~s}$, as measured in the simultaneous MECS data) in the PDS data, but the result was not conclusive, giving only a $3 \sigma$ upper limit of $50 \%$ to the pulsed fraction of a sinusoidal periodicity, to be compared to the $\sim 20 \%$ pulsed fraction observed below $10 \mathrm{keV}$.

Although we cannot rule out contamination from unknown transient sources, the flux measured in observation
Table 2. Status of the three transient sources within the PDS field of view during the BeppoSAX observations. The presence of the two $\mathrm{X}$-ray pulsars $(4 \mathrm{U} 1907+97$ and XTE J1906+09) is confirmed by the presence of their pulsations in the PDS data, while the black hole candidate (XTE J1908+94) by its detection in the MECS and LECS images and in the RossiXTE ASM lightcurve.

\begin{tabular}{|c|c|c|c|}
\hline Obs & $4 \mathrm{U} 1907+97$ & XTE J1906+09 & XTE J1908+94 \\
\hline $\mathrm{A}$ & OFF & OFF & OFF \\
\hline B & ON & ON & $\mathrm{OFF}$ \\
\hline $\mathrm{C}$ & $\mathrm{OFF}$ & $\mathrm{ON}$ & $\mathrm{OFF}$ \\
\hline $\mathrm{D}$ & $\mathrm{ON}$ & ON & OFF \\
\hline $\mathrm{E}$ & $\mathrm{OFF}$ & ON & $\mathrm{OFF}$ \\
\hline $\mathrm{F}$ & ON & $\mathrm{ON}$ & $\mathrm{OFF}$ \\
\hline $\mathrm{G}$ & OFF & ON & $\mathrm{ON}$ \\
\hline
\end{tabular}

A up to $\sim 150 \mathrm{keV}$ is most likely due to SGR $1900+14$ and therefore hereafter we assume that the spectral properties discussed in the text are associated to SGR $1900+14$ alone.

\subsection{Spectral analysis}

The background subtracted PDS spectrum of observation A can be well fit by a power-law with photon in$\operatorname{dex} \Gamma=1.6 \pm 0.3$, significantly flatter than that measured by INTEGRAL $(\Gamma=3.1 \pm 0.5$, see Fig. 3 ) in $2003 / 2004$. The corresponding 20-100 keV flux is $6 \times 10^{-11} \mathrm{erg} \mathrm{cm}^{-2} \mathrm{~s}^{-1}$, a factor $\sim 4$ higher than during the INTEGRAL observations, which confirms that before the giant flare the hard X-ray tail of SGR $1900+14$ was brighter.

The INTEGRAL spectrum was collected during $\sim 2.5 \mathrm{Ms}$ of different observations performed between March 2003 and June 2004, and thus it represents the hard X-ray emission of SGR $1900+14$ averaged over that long time period. Therefore, its relation to the soft $\mathrm{X}_{-}$ ray spectrum can be studied only comparing the spectra taken by other instruments in a similar time period, as shown for example in Fig. 3. The PDS instrument, instead, being a high sensitivity hard X-ray detector coupled to the MECS and LECS soft X-ray cameras, gives us the chance to study the broad band spectrum of SGR $1900+14$ during a single observation. Fitting the 1-150 keV BeppoSAX spectrum of observation A, we obtain a good result $\left(\chi^{2}=1.17\right.$ for 136 degrees of freedom) simply extrapolating to higher energies the best-fit model found in the soft $\mathrm{X}$-ray range. In fact, a fit with an absorbed power-law plus blackbody model gives the following parameters: photon index $\Gamma=1.04 \pm 0.08$, blackbody temperature $k_{B} T=0.50 \pm 0.06$, radius $R_{b b}=5 \pm 2 \mathrm{~km}$, and absorption $n_{\mathrm{H}}=(1.8 \pm 0.5) \times 10^{22} \mathrm{~cm}^{-2}$. 


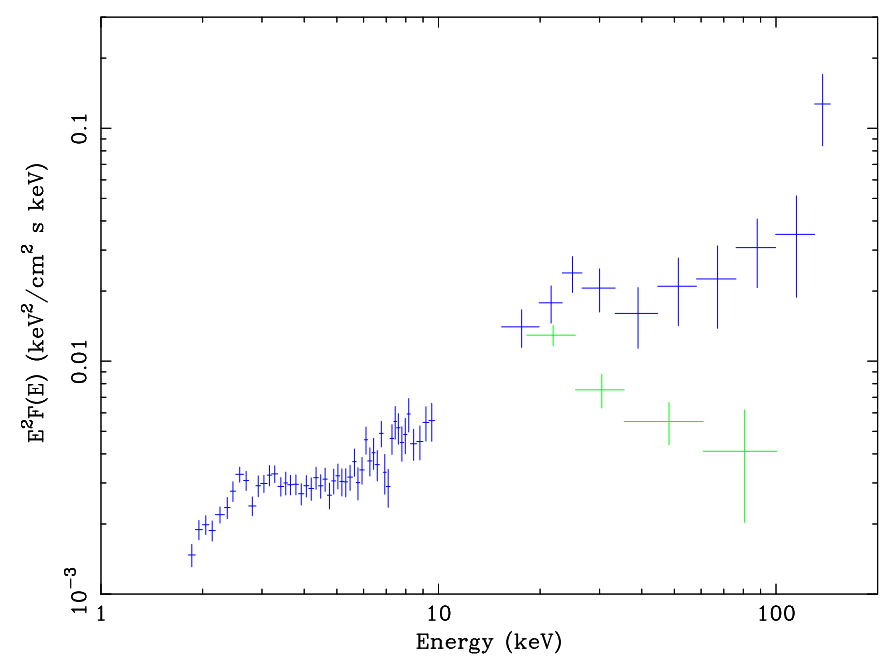

Fig. 3. Blue points: broad band spectrum of SGR 1900+14 taken on 1997 May 12 (observation A) with BeppoSAX (both MECS and PDS data). Green points: INTEGRAL data from March 2003 to June 2004.

\section{Conclusions}

We have studied the variability of SGR $1900+14$, both in the hard and in the soft $\mathrm{X}$-ray range, finding the following results:

- Except for the observations immediately following exceptional flares, the flux level in soft X-rays was stable while the source was moderately active and progressively decreased when it entered a 3 years long quiescent period.

- The intermediate flare of 2001 April 18 was followed by an X-ray afterglow that can be interpreted as due to the heating of a significant fraction of the neutron star surface, that then cools down in $\sim 1$ day. This is consistent with the interpretation of similar events in other magnetar candidates (Woods et al. 2004).

- The soft X-ray spectrum during the only available pre giant flare observation was harder than in the following quiescent observation. This is similar to what observed in SGR 1806-20, the only other SGR that could be monitored before and after a giant flare (Mereghetti et al. 2005; Tiengo et al. 2005).

- Comparing the hard X-ray spectrum of SGR 1900+14 recently observed with INTEGRAL to that observed with the PDS instrument in 1997, we find evidence for variations in flux and spectral slope.

- The reduction of the X-ray tail in coincidence with the giant flare is supported by the count rates detected in the PDS instrument above $50 \mathrm{keV}$ during the different BeppoSAX observations, that indicate how they significantly decreased already in the first post-flare observation. Since the hard X-ray tail in the spectrum of SGR 1900+14 might contain most of its total emitted energy, its variability in relation to the bursting activity is a key point to try to understand the SGR emission processes.

Acknowledgements. We gratefully acknowledge the support by the WE-Heraeus foundation.

\section{References}

Boella, G., Chiappetti, L., Conti, G., et al. 1997, A\&AS, 122,327

Feroci, M., Mereghetti, S., Woods, P., et al. 2003, ApJ, 596,470

Frontera, F., Costa, E., dal Fiume, D., et al. 1997, A\&AS, 122,357

Götz, D., Mereghetti, S., Tiengo, A., \& Esposito, P. 2006, A\&A, 449, L31

Guidorzi, C., Frontera, F., Montanari, E., et al. 2004, A\&A, 416, 297

Hurley, K., Cline, T., Mazets, E., et al. 1999a, Nature, 397,41

Hurley, K., Li, P., Kouveliotou, C., et al. 1999b, ApJ, 510, L111

in't Zand, J. J. M., Miller, J. M., Oosterbroek, T., \& Parmar, A. N. 2002, A\&A, 394, 553

Liu, Q. Z., van Paradijs, J., \& van den Heuvel, E. P. J. 2000, A\&AS, 147, 25

Mazets, E. P., Golenetskii, S. V., \& Guryan, Y. A. 1979, Soviet Astronomy Letters, 5, 343

Mereghetti, S., Chiarlone, L., Israel, G. L., \& Stella, L. 2002, in Neutron Stars, Pulsars, and Supernova Remnants, ed. W. Becker, H. Lesch \& J. Trümper, [ArXiv: astro-ph/0205122]

Mereghetti, S., Esposito, P., Tiengo, A., et al. 2006, ApJ, in press (astro-ph/0608588)

Mereghetti, S., Tiengo, A., Esposito, P., et al. 2005, ApJ, 628, 938

Parmar, A. N., Martin, D. D. E., Bavdaz, M., et al. 1997, A\&AS, 122, 309

Thompson, C. \& Duncan, R. C. 1995, MNRAS, 275, 255

Thompson, C. \& Duncan, R. C. 1996, ApJ, 473, 322

Tiengo, A., Esposito, P., Mereghetti, S., et al. 2005, A\&A, 440, L63

Woods, P. M., Kaspi, V. M., Thompson, C., et al. 2004, ApJ, 605, 378

Woods, P. M., Kouveliotou, C., van Paradijs, J., Finger, M. H., \& Thompson, C. 1999, ApJ, 518, L103

Woods, P. M. \& Thompson, C. 2004, in "Compact Stellar X-ray Sources", ed. W.H.G. Lewin and M. van der Klis, [ArXiv: astro-ph/0406133] 